\title{
The Features of Low Frequency Atomic Vibrations and Propagation of Acoustic Waves in Heterogeneous Systems
}

\author{
Alexander Feher ${ }^{1}$, Eugen Syrkin², Sergey Feodosyev², Igor Gospodarev², \\ Elena Manzhelii'2, Alexander Kotlar ${ }^{2}$ and Kirill Kravchenko ${ }^{2}$ \\ ${ }^{1}$ Institute of Physics, Faculty of Science P. J. Šafárik University, Košice \\ ${ }^{2}$ B.I.Verkin Institute for Low Temperature Physics and Engineering NASU, Kharkov \\ ${ }^{1}$ Slovakia \\ ${ }^{2}$ Ukraine
}

\section{Introduction}

In recent years, the quasi-particle spectra of various condensed systems, crystalline as well as disordered and amorphous, became also the "object" of applications and technical developments and not only of fundamental research. This led to the interest in the theoretical and experimental study of the quasi-particle spectrum of such compounds, which are among the most popular and advanced structural materials. Most of these substances have heterogeneous structure, which is understood as a strong spatial heterogeneity of the location of different atoms and, consequently, the heterogeneity of local physical properties of the system, and not as the coexistence of different phases (i.e. heterophase). To these structures belong disordered solid solutions, crystals with a large number of atoms per unit cell as well as nanoclusters.

This chapter is devoted to the study of vibration states in heterogeneous structures. In such systems, the crystalline regularity in the arrangement of atoms is either absent or its effect on the physical properties of the systems is weak, affecting substantially the local spectral functions of different atoms forming this structure. This effect is manifested in the behavior of non-additive thermodynamic properties of different atoms (e.g. mean-square amplitudes of atomic displacements) and in the contribution of individual atoms to the additive thermodynamic and kinetic quantities. The most important elementary excitations appearing in crystalline and disordered systems are acoustic phonons. Moreover, in heterogeneous nanostructures the application of the continuum approximation is significantly restricted; therefore we must take into account the discreteness of the lattice. This chapter contains a theoretical analysis at the microscopic level of the behavior of the spectral characteristics of acoustic phonons as well as their manifestations in the lowtemperature thermodynamic properties.

The chapter consists of three sections. The first section contains a detailed analysis at the microscopic level of the propagation of acoustic phonons in crystalline solids and disordered solid solutions. We analyze the changes of phonon spectrum of the broken crystal regularity of the arrangement of atoms in the formation of a disordered solid 
solution with heavy isotope impurities and randomly distributed impurities weakly coupled both with the atoms of the host lattice and among themselves. As is well known, such defects enrich the low-frequency phonon spectrum and lead to a significant change in the low-temperature thermodynamic and kinetic characteristics (see, for example, Kosevich, 1999; Maradudin et al., 1982; Lifshitz, 1952a). In particular, the impurity atoms cause the socalled quasi-localized vibrations (Kagan \& Iosilevskij, 1962; Peresada \& Tolstoluzhskij 1970, 1977; Cape et al., 1966; Manzhelii et al., 1970). This section analyzes in detail the conditions of the formation and evolution of quasi-localized vibrations with increasing concentration of impurities. It is shown that the quasi-local maximum in the phonon spectrum of the lowfrequency zone is formed by vibrations localized on impurity atoms. Rapidly propagating phonons corresponding to the vibrations of the host lattice atoms are scattered by localized vibrations. This scattering forms a kink in the local spectral densities of these atoms that is similar in shape to the first van Hove singularity of a perfect crystal. In the description of the spectral characteristics of the elementary excitations in heterogeneous structures such theoretical methods are necessary which do not involve the translational symmetry of the crystal lattice. In this section we use such a method for computing the local Green's functions and the local and partial spectral densities.

These self-averaging spectral characteristics (Lifshitz et al., 1988) can be determined also for compounds that do not possess regularity in their crystal structure. An effective method for describing disordered systems and calculating their quasi-particle spectra is the method of Jacobi matrices ( $J$-matrices) (Peresada, 1968; Peresada et al., 1975, Haydock et al., 1972). By this method the majority of the calculations in this paper were carried out.

The second section is devoted to the analysis of the reasons for the strong temperature dependence of the Debye temperature $\Theta_{D}(T)$ under $T \leq 0.1 \Theta_{D}$. The temperature dependence $\Theta_{D}(T)$ is a solution of the transcendental equation $C_{D}\left(T / \Theta_{D}\right)=C(T)$, where $C(T)$ is calculated at the microscopic level or experimentally determined and $C_{D}\left(T / \Theta_{D}\right)$ is the temperature dependence of the Debye heat capacity. It is shown that the reason for the formation of a low-temperature minimum on the dependence $\Theta_{D}(T)$ are the fast-propagating lowfrequency phonons (propagons) (Allen et al., 1999) scattered on the slow quasi-particles. In the case of a defect (random reduction of force constants) the quasi-localized vibrations do not form, but in the ratio of the phonon density of states $v(\omega)$ to the square of the frequency a maximum in the propagon zone of the phonon spectrum is formed with increasing concentration of defects. The maxima in the ratio $v(\omega) / \omega^{2}$ are called boson peaks (see, for example, Feher et al., 1994; Gurevich et al., 2003; Schrimacher et al., 1998). They are intensively studied for systems with topological disorder, glasses and such compounds as molecular crystals with rotational degrees of freedom. In this section we analyze the arising of such features in solid solutions with only vibration degrees of freedom. The frequency of the boson peak coincides with the frequency of the quasi-local vibrations corresponding to a weakly bound impurity at concentrations, for which the average distance between the randomly distributed impurity atoms corresponds to the propagon frequency equal to the frequency of quasi-local vibrations. That is, the distance between the impurities (disorder parameter) becomes comparable to the wavelength of rapid acoustic phonons with the frequency equal to the quasi-local vibration frequency. This corresponds to the phonon Ioffe-Regel crossover (Klinger \& Kosevich, 2001, 2002). It is shown that the temperaturedependence and magnitude of $\Theta_{D}(T)$ are even more informative than the phonon density of states. It was shown on the example of a $\mathrm{Kr}_{1-\mathrm{p}} \mathrm{Ar}_{\mathrm{p}}$ solid solution that for $p \approx 25 \%$ there are no singularities both in the propagon zone of the phonon density of states and in the phonon 
density relation to the square of frequency. At the same time, the temperature dependence of the relative (compared with pure $\mathrm{Kr}$ ) changes in the low-temperature heat capacity shows two peaks that can not be explained by the superposition of contributions of isolated impurities, impurity pairs, etc. The reason of this behavior is the scattering of fast propagating phonons, corresponding to the krypton atoms, on significantly slower phonons corresponding to the vibrations of atoms in argon clusters.

Many features of the phonon spectra and vibrational characteristic of disordered heterogeneous structures are also inherent to the crystals with polyatomic unit cells. Third section of this work is devoted to the analysis of phonon spectra and vibrational characteristics of such crystals. The manifestations of the phonon Ioffe-Regel crossover in multilayered regular crystalline structures are analyzed. The presence of the quasi-twodimensional and quasi-one-dimensional features in the behavior of the vibrational characteristics of multilayer compounds is shown. The macroscopic characteristics of such compounds are derived from the low-dimensional ones. This allows us to describe the vibrational characteristics of such complex compounds in frames of low-dimensional models. The features of the interaction of phonons with a planar defect are investigated using these models. In particular, the resonance effects in the scattering of acoustic waves and the formation of localized and resonance vibrational states in the planar defect are considered. Such effects may lead to singularities in the experimentally observed kinetic characteristics of the grain boundaries. The heat transfer between two different media, on the condition that the Fano resonance occurs, is analyzed.

\section{Low-frequency characteristics of the phonon spectra of disordered solid solutions}

This chapter is devoted to the study of the propagation of acoustic phonons at different frequencies of quasi-continuous FCC crystal phonon spectrum. We analyze in more detail the analogy of the Van Hove singularity in the phonon spectrum of the perfect crystal with similar features of the phonon spectra of structures with broken regularity in the arrangement of atoms of a crystal. For any solid (both crystal and the one which does not possess the translational symmetry of the atoms arrangement), a low-frequency range exists where the dispersion relation of phonons has the form $\omega(\boldsymbol{k})=s(\boldsymbol{\kappa}) k$ ( $k$ is a module of the wave vector $\boldsymbol{k}, \boldsymbol{\kappa} \equiv \boldsymbol{k} / \boldsymbol{k}$, and $s(\boldsymbol{\kappa})$ is the velocity of sound). The phonon density of states in this range takes the Debye form $v(\omega) \sim \omega^{2}$. With the increase of the $k$-value the phonon dispersion relation increasingly deviates from the linear one (frequency $\omega$ becomes lower than sk) and the actual density of states deviates upwards from the Debye one. At low frequencies, the sound propagation occurs along all crystallographic directions. With increasing frequency the propagation velocity of acoustic phonons decreases, this decrease being different for different crystallographic directions. In a perfect crystal, when the phonon frequency corresponds to the frequency of the first van Hove singularity $\omega=\omega^{*}$, the propagation of the transverse sound along one of the crystallographic directions (in the FCC it is the crystal direction $\Gamma L$, Fig. 1a) ceases and the corresponding group velocity is zero. Phonons with frequencies $\omega<\omega^{*}$ were named propagons and those with higher frequencies are called diffusons. With a further frequency increase also the number of directions increases along which the propagation of sound ceases. The highest frequency of the van Hove singularity $\left(\omega=\omega^{* *}\right)$ corresponds to the frequency at which the wavelength 
of the longest wavelength phonons is smaller than the interatomic distance (Fig. 1b). Phonons with $\omega>\omega^{* *}$ are almost localized and they were named locons, while the frequency interval $\left(\omega^{* *}, \omega_{m}\right)$ is called the locon band.
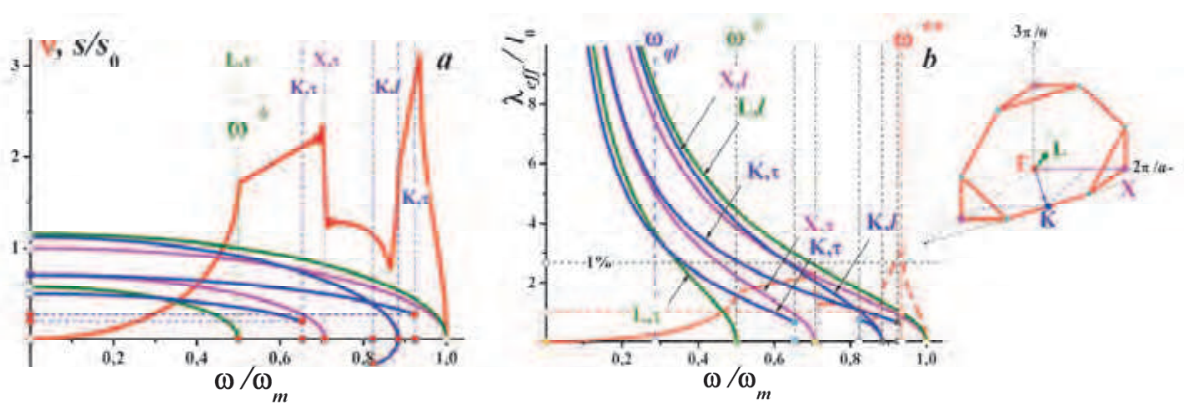

Fig. 1. The phonon density of states (red lines), the frequency dependences of the group velocities of phonon modes (part a) and frequency dependences of the values $\lambda_{\text {eff }}(\omega) / l_{0}$ (part $\mathbf{b}$ ) along the main of the highly symmetrical crystallographic directions of FCC crystal with central nearest-neighbors interaction (blue, purple and olive line, depending on the direction). The first octant of the first Brillouin zone of a FCC crystal with indications of considered high-symmetry directions is shown on the right $\left(l_{0} \equiv a / \sqrt{2}\right.$ is the distance between nearest neighbors).

Note that the propagation character of locons and diffusons practically does not differ from the propagation character of optical phonons in a crystal with complex lattice. A translational symmetry disturbance does not lead to a qualitative modification in the nature of acoustic phonons and does not change their classification.

Let in the crystal randomly introduce heavy isotopic substitution impurities or impurities weakly coupled to the atoms of the host lattice and among themselves. Neglecting the interaction between impurities the variations of the phonon spectrum are satisfactorily described by the theory of regular perturbations devised by I.M. Lifshitz (Lifshitz, 1952a). In particular, the formation of the so-called quasi-local vibrations (QLV) due to the presence of heavy or weakly bound impurities was predicted (Kagan \& Iosilevskij, 1962) and studied in detail both theoretically (see, for example, Peresada \& Tolstoluzhskij 1977) and experimentally (see, for example, Cape et al., 1966; Manzhelii et al., 1970). The QLV are manifested by the resonance peaks in the low-frequency part of the phonon spectrum and they contribute essentially to the low-temperature thermodynamic properties. At low impurity atoms concentrations $p<<1$, the vibration characteristics of the solid solution can be described within the linear in $p$ approximation:

$$
\tilde{v}(\omega)=v(\omega)+p \sum_{i} \Delta \rho^{(i)}(\omega)
$$

The summation is performed over all cyclic subspaces (Peresada, 1968; Peresada et al.,1975), in which the operator $\hat{\Lambda}$ describing the perturbation of the lattice vibrations by either isolated heavy or weakly coupled impurity is non-zero, $\Delta \rho^{(i)}(\omega)$ is the spectral density 
change in each of these subspaces, $\tilde{v}(\omega)$ and $v(\omega)$ are the phonon densities of solid solution and perfect crystal states, respectively. If in each of the cyclir surhsnaces the operator $\hat{\Lambda}$ induces a regular degenerate operator, then the value $\Delta \rho^{(i)}(\omega)$ can be calculated using the spectral shift function $\xi(\omega)$ (Lifshitz, 1952a). Using the expressions obtained for this function in the J-matrix method (Peresada, 1968; Peresada et al., 1975, Peresada \& Tolstoluzhskij 1977), we obtain:

$$
\Delta \rho(\omega)=-\frac{d \xi(\omega)}{d \omega}=\frac{\rho^{2}(\omega)}{\pi^{2} \rho^{2}(\omega)+[S(\omega)-\operatorname{Re} G(\omega)]^{2}} \cdot \frac{d}{d \omega}\left[\frac{S(\omega)-\operatorname{Re} G(\omega)}{\rho(\omega)}\right]
$$

where the function $S(\omega)$ describes the perturbation by defect and depends on the defect parameters, $G(\omega)$ is the local Green's function of a perfect crystal. If in any cyclic subspace the solution of the equation

$$
S(\omega)-\operatorname{Re} G(\omega)=0
$$

is $\omega=\omega_{k}$, then in the vicinity of this value the expression (2) has a resonant character:

$$
-\frac{d \xi}{d \omega}=\frac{2}{\pi} \frac{\Gamma}{4\left(\omega-\omega_{k}\right)^{2}+\Gamma^{2}} ; \quad \Gamma \equiv \frac{\pi \rho(\omega)}{\frac{d}{d \omega}[S(\omega)-\operatorname{Re} G(\omega)]_{\omega=\omega_{k}}}
$$

The equation (3) formally coincides with the Lifshitz equation which yields (of course for other values of $S(\omega)$ ) the frequencies of discrete vibrational levels, lying outside the band of quasi-continuous spectrum of the crystal (Lifshitz, 1952a). However, these discrete levels are, in contrast to the values $\omega_{k}$, the poles of the perturbed local Green's function. The Green's function can not have poles within the quasi-continuous spectrum. The possibility to determine the QLV frequencies using equation (3) arises from the fact that at low frequencies $\left|\operatorname{Re} G_{00}(\omega)\right| \gg>\operatorname{Im}_{00}(\omega)$.

Let us analyze the quasi-local oscillations due to the substitution impurity in an FCC crystal with the central interaction of nearest-neighbors. The interaction of the impurity with the host lattice is also considered as a purely central and, therefore, the perturbation caused by such an impurity should be regular and degenerate. Let us consider two cases: the isotopic impurity with a mass four times higher than that of the host lattice (i.e. the mass defect is $\varepsilon \equiv \Delta m / m=3$ ) and the impurity atom with a mass equal to the mass of a host lattice atom $\varepsilon=0$ and coupled to the host lattice four times weaker than are the atoms of the host lattice between each other $(v \equiv \Delta \alpha / \alpha=-3 / 4$ is the coupling defect). In the first case, operator $\hat{\Lambda}$ induces a non-zero operator only in the cyclic subspace which is generated by the displacement of the impurity atom. The vectors corresponding to this subspace transform according to the irreducible representation $\tau_{-}^{5}$ of the symmetry group of the lattice $O_{h}$ (the notation of Kovalev, 1961). In the given subspace the spectral density of perfect lattice coincides with its density of states. For an isotopic impurity the function $S(\omega)$ (Peresada \& Tolstoluzhskij, 1977) reads:

$$
S_{i s}(\omega)=-\frac{2}{\omega \varepsilon}
$$


In the second case, except the subspace $H^{\left(\tau_{-}^{5}\right)}$ where the function $S(\omega)$ is

$$
S_{w}^{\left(\tau_{-}^{5}\right)}(\omega)=\frac{2}{\omega}+\frac{\omega_{m}^{2}(1+v)}{\omega^{3} v}
$$

the non-zero operators will be operators induced by the operator $\hat{\Lambda}$ in cyclic subspaces transformed according to irreducible representations, $\tau_{+}^{1}, \tau_{+}^{3}, \tau_{+}^{4}$ and $\tau_{-}^{4}$ of the same group $O_{h}$. Over all of these four subspaces $S(\omega)=16 \omega /\left(\omega_{m}^{2} v\right)$. For weakly bound impurity, the function $S_{w} \leq S_{\lim }=-16 \omega / \omega_{m}$ and equation (3) can not have solutions in the propagon zone within these cyclic subspaces. Therefore, for real values of parameter $v$ equation (3) has a solution in the subspace $H^{\left(\tau_{-}^{5}\right)}$ only. This solution for both cases shows Fig. 2 . The real part of the Green's function (curves 2 in both parts) crosses the dashed curves 3, which represent the equations (5) (part $\boldsymbol{a}$ ) and (6) (part $\boldsymbol{b}$ ), at points $\omega_{k}$. This figure also shows the spectral densities $\rho^{\left(\tau_{-}^{5}\right)}(\omega)$ of the perfect crystal, coinciding with its phonon density of states $v(\omega)$ (dashed curves 1), and phonon densities of states of the corresponding solid solutions with concentration $p=5 \%$. This figure shows the phonon density of states (curves 1 ) for both the heavy isotopes (part $\boldsymbol{a}$ ) and weakly bound impurities (part $\boldsymbol{b}$ ). Curves 4 show the contributions from impurities and curves 6 those from the matrix lattice. We can see that the maxima formed on the phonon densities $\tilde{v}(\omega, p)$ are (curves 1) completely caused by the vibrations of impurity atoms. Let us analyze figures 2 and 3 together. The value of the phonon density of states of a perfect crystal at $\omega=\omega_{k}$ can not be considered as negligible, since it is comparable to the value of the real part of the Green's function at this frequency $\left(v\left(\omega_{k}\right) \sim 0.1 \operatorname{Re} G\left(\omega_{k}\right)\right)$. Therefore, as is seen from the figures, though the frequencies of the maxima on the curves $\tilde{v}(\omega, p)$ and $v_{\text {imp }}(\omega, p)$ are close to the frequency $\omega_{k}$, they do not coincide with it (Fig. 2b) (especially in the case of a weakly bound impurity). For weakly bound impurity one should expect a higher degree of localization of QLV on impurity atoms. In Fig. 3 the values of $v_{\text {imp }}(\omega, p)$ are compared with the spectral density of isolated impurity atoms $\tilde{\rho}^{\left(\tau_{-}^{5}\right)}(\omega)=\frac{2 \omega}{\pi} \operatorname{Im}\left(\vec{h}_{0}^{\left(\tau_{-}^{5}\right)},\left[\omega^{2} \hat{I}-\widehat{L}-\hat{\Lambda}\right]^{-1} \vec{h}_{0}^{\left(\tau_{-}^{5}\right)}\right)$. The function $v_{i m p}(\omega, p)$ is nonzero only near frequencies $\omega_{q l}$, which are the maxima on curves 5 . Therefore the frequency $\omega_{q l}$ can be more reasonably than $\omega_{k}$ considered as the frequency of QLV (quasilocal frequency).

Therefore, QLV can be represented as waves slowly diverging from the impurity, similar to spherical waves. Fig. 2 also presents (curves 4 ) the values of $\rho_{01}^{\left(\tau_{-}^{5}\right)}(\omega)$, i.e. the spectral correlators of displacements of impurity atoms with their first coordination sphere

$$
\rho_{01}^{\left(\tau_{-}^{5}\right)}(\omega)=\frac{2 \omega}{\pi} \operatorname{Im}\left(\vec{h}_{1}^{\left(\tau_{-}^{5}\right)},\left[\omega^{2} \hat{I}-\hat{L}-\hat{\Lambda}\right]^{-1} \vec{h}_{0}^{\left(\tau_{-}^{5}\right)}\right)=P_{1}\left(\omega^{2}\right) \rho^{\left(\tau_{-}^{5}\right)}(\omega)
$$



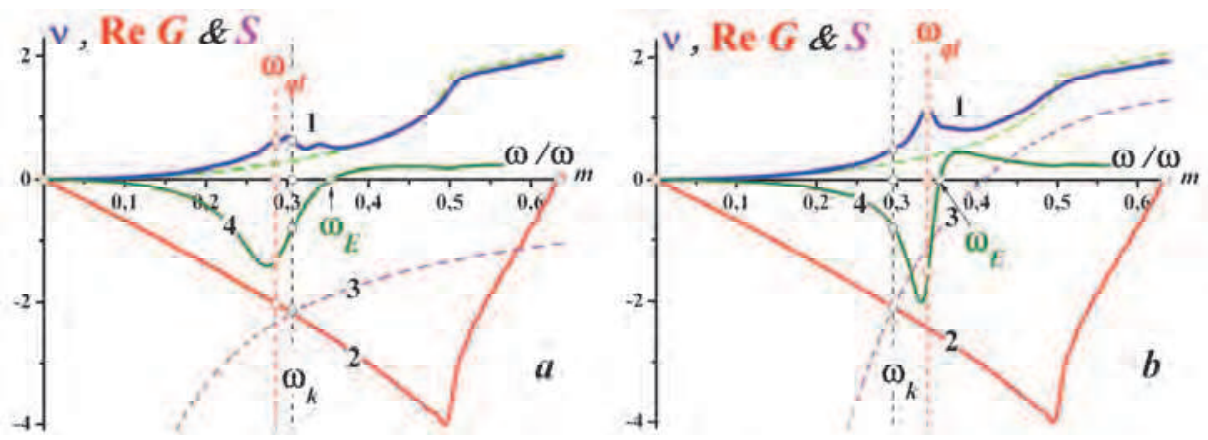

Fig. 2. Phonon densities of disordered solid solutions with impurity concentration $5 \%$ (curves 1) and solutions of the equation (3) (intersection of curves 2 and 3) for cases of a heavy isotopic impurity (part a) and weakly bound impurities (part b). Curves 4 in both parts are spectral correlators of vibrations of the impurity atom with its first coordination sphere.

where $P_{1}\left(\omega^{2}\right)$ is the polynomial defined by the recurrence relations for the J-matrix of the perturbed operator $\hat{L}+\hat{\Lambda}$ (Peresada, 1968; Peresada et al.,1975). Spectral correlator $\rho_{01}(\omega)$ vanishes when $\omega=\omega_{E}$, where $\omega_{E}$ is the Einstein frequency of the correspondent subspace $\left(\omega_{E}^{2}=\int_{0}^{\omega_{m}} \omega^{2} v(\omega) d \omega\right)$. Thus, when $\omega=\omega_{E}$, the correlation with the first coordination sphere is absent, and the close is the frequency $\omega_{q l}$ to $\omega_{E}$, the stronger is the degree of localization of QLV. As it could be seen from Fig. 2 the QLV frequency for weakly bound impurity is nearly three times closer to $\omega_{E}$ than that the heavy isotopic one, and the quasi-local maximum for a weakly bound impurity has a sharper resonance form than the maximum for a heavy isotopic defect.

The QLV are localized near the impurity atoms and their formation is very similar to the occurrence of discrete vibrational levels (local oscillations) outside the continuous spectral band of the host lattice in the presence of light or strongly coupled impurities in a crystal. However, there is an important fundamental difference between the local and quasi-local vibrations, manifested under increasing concentration of impurity atoms. Local vibrations are the poles of the Green's function of the perturbed crystal, and their amplitudes decay exponentially with the distance from the impurity. Being located outside the quasicontinuous spectrum, these vibrations do not interact with the phonon modes of the host lattice. With an increasing concentration of either light or strongly coupled impurities their effect upon phonon spectrum can be determined by taking into account the expansion of concentration (Lifshitz et al., 1988). Thus, the increase of the concentration of light impurities leads to the appearance of sharp resonant peaks in phonon spectrum with frequencies coincident with those of local vibrations of the isolated impurity atom pairs and eventually, regular triangles and tetrahedrons (Kosevich et al., 2007). The QLV are not the poles of the Green's function, they are common non-divergence maxima in the phonon density of states. Though, as is shown in the next section, these peaks are formed by the impurity atoms vibrations which interact with the phonon modes of host lattice. Therefore, at finite (even low enough, about few percents) concentrations of heavy or weakly coupled impurity atoms, the significant modification of the entire phonon spectrum of the crystal occurs, 

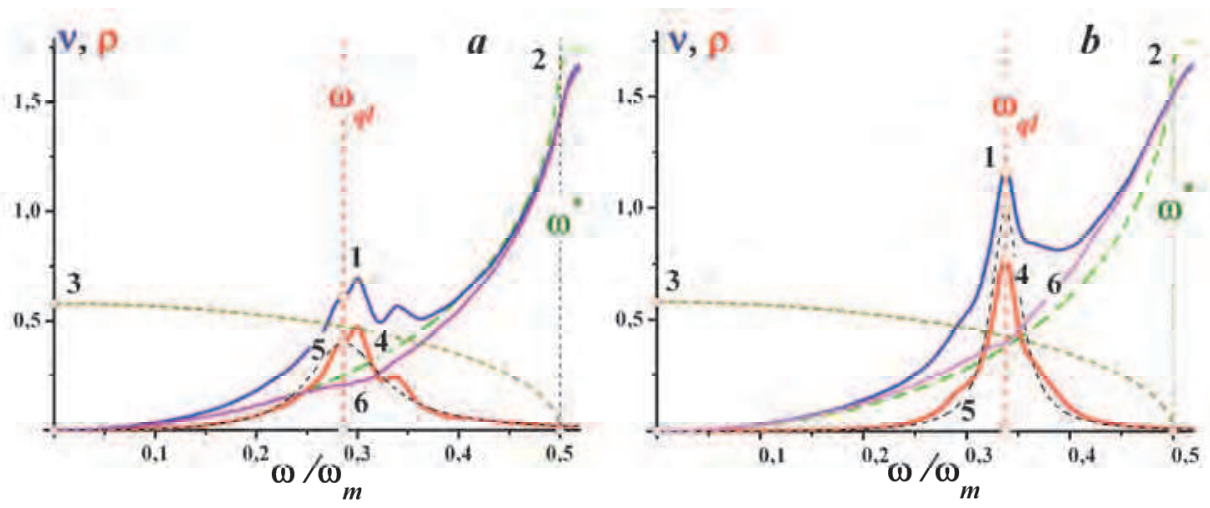

Fig. 3. Phonon densities of disordered solid solutions with impurity concentration $5 \%$ (curves 1); from contributions impurity atoms (curves 4 ) and atoms of the host lattice (curves 6). Part a corresponds to the heavy isotopic impurity $\left(\mathrm{m}^{\prime} / \mathrm{m}=4\right)$, part $\mathbf{b}$ corresponds to a weakly bound impurity $\left(\alpha^{\prime} / \alpha=1 / 4\right)$. Curves 2 show the phonon density of the original perfect lattice, curves 3 represent the frequency dependence of the transverse sound velocity along the crystallographic direction $\Gamma L$, and curves 5 show the spectral density of single isolated impurities, multiplied by the concentration $p=0.05$.

which can not be described by the expansion of the impurity concentration. Thus the weakening of bonds between the argon impurities in krypton matrix leads to a characteristic "two-extreme" behavior of the temperature dependence of the relative change in the lowtemperature heat capacity unexplained by the superposition of contributions of isolated impurities, impurity pairs, triples and etc., without taking into account the restructuring of the entire spectrum (Bagatskii et al., 2007). The restructuring of the phonon spectrum of the crystal and the delocalization of QLV at finite concentrations of impurities in the coherent potential approximation was considered in (Ivanov, 1970; Ivanov \& Skripnik, 1994).

The QLV usually occur in the frequency range where corresponding wavelengths of acoustic phonons of the host lattice become comparable to the average distance between the defects (the so-called disorder varameter). This is valid even for low concentrations of impurity atoms as is illustrated in Fig. $1 b$. The value $\lambda\left(\omega_{q l}\right)$ for most phonon modes exceeds the disorder parameter even at $p \approx 1 \%$. Therefore an interaction of QLV with rapidly propagating acoustic phonons of the host lattice (propagons) appears as the IoffeRegel crossover as is shown in (Klinger \& Kosevich, 2001, 2002) and can lead to the formation of a boson peak (BP). The BP is an anomalous override of the low-frequency phonon density over the Debye density. The BP was observed in the Raman and Brillouin scattering spectra (Hehlen et al., 2000; Rufflé et al., 2006) and in inelastic neutron scattering experiments (Buchenau et al., 1984) as maxima in the frequency dependence $v(\omega) / \omega^{2}$ or $I(\omega) / \omega^{2}(I(\omega)$ is the scattering intensity). These peaks appear in the low-frequency region (between 0.5 and $2 \mathrm{THz}$ ) of the vibration density of states (Ahmad et al., 1986), i.e. far below the Debye frequency. At the BP frequency the transition occurs from the fast-propagating low-frequency phonons (propagons), with dispersion relation close to the acoustic one, to 
the so-called diffusons, i.e. phonons, whose propagation is hampered by the scattering on localized states (Feher et al., 1994). In the frequency range $\left(\omega^{*}, \omega^{* *}\right)$, the number of localized vibrations increases with the frequency increase (Fig. 1a). Therefore, the phonons with frequencies lying in that interval (diffuson area) are either diffusons or locons. The similarity of the boson peak in disordered systems (e.g. glasses and substitution solid solutions) to the first van Hove singularity in crystal structures is noted in (Buchenau et al., 2004; Gospodarev et al., 2008). BPs are also observed in polymeric and metallic glasses (Duval et al., 2003; Arai et al., 1999).

As is shown in Fig.4 in the frequency range $\left[0, \omega^{*}\right]$ the vibrations of atoms of the host lattice propagate rapidly and are scattered by the quasi-localized states formed by impurity vibrations. Curves 6 in this figure depict the frequency dependence $\tilde{v}(\omega, p)-v_{\text {imp }}(\omega, p)$. At frequencies $\omega<\omega_{q l}$ there vibrations propagate as plane waves. Corresponding parts of curves 6 are smooth and have parabolic (quasi-Debye) form. At $\omega \approx \omega_{q l}$ a kink similar to the shape of the first van Hove singularity can be seen on curves 6. At this frequency as well as at $\omega=\omega^{*}$ in the phonon spectrum of a perfect crystal (Fig. 1a) there is a sharp change of the average group velocity of phonons. The frequency $\omega_{q l}$ is the upper limit of propagon zone of solid solution. This is clearly exhibiting with increasing impurity concentration p. Fig. 4 shows the evolution of the contribution to the phonon density of states by the displacements of impurity atoms (part a) and by the displacements of atoms of the host lattice (part $\mathbf{b}$ ) with increasing impurity concentration. Note that both dependences can be determined experimentally (e.g., by the method described in (Fedotov et al., 1993). On both parts of Fig. 4 dashed curves show phonon densities of states of the perfect host lattice. In addition, functions $p \tilde{\rho}^{\left(\tau_{-}^{5}\right)}(\omega)$ are depicted in Fig. $4 \mathbf{a}$ by dashed lines. It is seen that at concentrations $p \in[0.1,0.5]$ the values of both $v_{\text {imp }}(\omega, p)$ and $p \tilde{\rho}^{\left(\tau_{-}^{5}\right)}(\omega)$ are different from zero in the same frequency range near the quasi-local frequency $\omega_{q l}$. For $\omega<\omega_{q l}$ the frequency dependence takes parabolic form (quasi-Debay form). The frequency dependences $\tilde{v}(\omega, p)-v_{\text {imp }}(\omega, p)$ also have a characteristic kink at $\omega \approx \omega_{q l}$, similar in shape to the first van Hove singularity (observed at all concentrations, even at $p=0.9$ ). That is at $p \leq 0.5$ the quasi-local frequency is an upper bound of the propagon zone for the vibrations of both impurity atoms and atoms of the host lattice.

With increasing concentration ( $p \geq 0.25$ ) a singularity of the kink type begins to form on the function $v_{\text {imp }}(\omega, p)$ at $\omega \approx \omega_{q l}$. At concentrations $p>0.5$ large enough impurity clusters are formed in the solid solution. There is a short-range order in such clusters and we can identify the different crystallographic directions. The structure consisting of such clusters can already be considered as a structure with topological disorder and for given values of the concentration the upper limit of the propagon zone corresponds to the vanishing of the group velocity of the transversally polarized phonons along the crystallographic direction $\Gamma L$ in impurity clusters. This frequency, as shown in Fig. $4 \mathbf{a}$, is lower than $\omega_{q l}$. With increasing $p$ it approaches to the value of the frequency of the first van Hove singularity of perfect crystal consisting of heavy impurity atoms $\tilde{\omega}^{*}$.

Thus the influence of impurity atoms, which are heavy or weakly bound to the atoms of host lattice, on the phonon spectrum and the vibrational characteristics is manifested both in the formation of quasilocal vibrations caused by the vibrations of impurities and in the scattering on these vibrations of fast acoustic phonons generated by atomic vibrations of the 


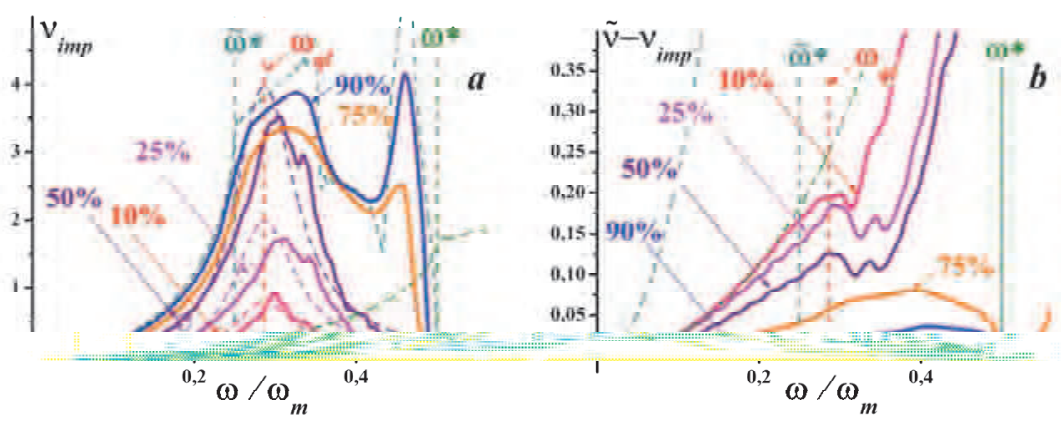

Fig. 4. Part a shows the evolution of function $v_{i m p}(\omega, p)$ with increasing concentration of impurities. Part $\mathbf{b}$ shows the evolution of function $\tilde{v}(\omega, p)-v_{i m p}(\omega, p)$ with increasing concentration of impurities.

host lattice. Up to concentrations of $p \approx 0.5$ the quasi-local frequency is an upper boundary of the propagon band, i.e. the frequency interval in which the phonons propagate freely in all directions. Further increase in the concentration is accompanied by the shift of the propagon zone upper boundary to the frequency of the first van Hove singularity of the crystal consisting of impurity atoms $\tilde{\omega}^{*}<\omega_{q l}$. At the same time for the propagation of atomic vibrations of the host lattice the upper boundary of the propagon zone is quasi-local frequency $\omega_{q l}$.

\section{Phonon spectra and low-temperature heat capacity of heterogeneous structures with bonds randomly distributed between atoms}

The Debye approximation widely used for the description of the thermal properties of solids is based on an approximation of the real vibrational spectrum of the crystal by phonons with acoustic dispersion law. The corresponding density of states is (see, e.g. Kosevich, 1999):

$$
v_{D}^{(q)}(\omega)=\frac{q \omega^{q-1}}{\omega_{D}^{q}} .
$$

Fig. 5 a shows the Debye density of states $v_{D}^{(3)}(\omega)=3 \omega^{2} / \omega_{D}^{3}$, defined by (9) at $q=3$ (curve 1), compared with the true density of states of the FCC lattice with central interaction of nearest neighbors (curve 2). It is seen that at $\omega \leq 0.25 \omega_{m}$ these curves almost coincide. With the frequency increase a deviation of the phonon density from $v_{D}^{(3)}(\omega)$ occurs. This leads to a deviation of the temperature dependence of the phonon heat capacity from its Debye form $C_{D}(T)$. Moreover, this deviation is more apparent the lower the frequencies are at which such deviation starts. As a rule, the deviation of the true phonon heat capacity from $C_{D}(T)$ is described as a temperature dependence of the Debye temperature $\Theta_{D}$. This dependence can be derived from the transcendental equation

$$
C_{v}(T)=C_{D}(T) \equiv 3 R\left\{D\left(\frac{\Theta_{D}}{T}\right)-\frac{\Theta_{D}}{T} D^{\prime}\left(\frac{\Theta_{D}}{T}\right)\right\} ; \quad D(x) \equiv \frac{3}{x^{3}} \int_{0}^{x} \frac{z^{3} d z}{e^{z}-1},
$$


where the heat capacity $C_{v}(T)$ is determined from experiment or microscopic calculation as

$$
C_{v}(T)=3 R \int_{0}^{\omega_{m}}\left(\frac{\hbar \omega}{2 k T}\right) \cdot \operatorname{sh}^{-2}\left(\frac{\hbar \omega}{2 k T}\right) \cdot v(\omega) d \omega .
$$

Of course, at $v(\omega)=v_{D}^{(3)}(\omega)$ the expressions (10) and (11) coincide and $\Theta_{D}(T)=\Theta_{P} \equiv \hbar \omega_{m} / k$, i.e. the Debye temperature does not depend on temperature. At low temperatures ( $T<<\Theta_{P}, \Theta_{D}$ ) the main contribution to the heat capacity is provided by the long-wavelength phonons with the sound dispersion relation. It seems that the dependence $C_{v}(T)$ is well described by (10). That is, the Debye temperature should be practically the same as $\Theta_{P}$. Indeed, as seen from Fig. $5 \mathbf{b}$ (curve 2), exactly in the temperature range $T \leq 0.1 \Theta_{P}$ the dependence $\Theta_{D}(T)$ is most intense. This is typical for a large number of compounds (Leibfried, 1955). To find the cause of a strong temperature dependence of $\Theta_{D}$ at $T<<\Theta_{D}$ we consider the function $\Theta_{D}(T)$ for a system for which the phonon density of states is a linear combination of the function $v_{D}^{(3)}(\omega)$ (curve 1 in Fig. 5a) and the Einstein density of states $\delta\left(\omega-\omega^{*}\right)$, where $\omega^{*}$ is the frequency of the first van Hove singularity (dashed line 3 in Fig. 5a). Curve 3 in Fig. $5 \mathbf{b}$ shows the $\Theta_{D}(T)$ in the case when the phonon density of states is $v_{\text {appr }}(\omega)=\frac{8}{39} \delta\left(\omega-\omega^{*}\right)+\frac{31}{39} v_{D}^{(3)}(\omega)$. The coefficients of this linear combination are selected from the averaging over all the high-symmetry directions in the FCC lattice. As shown in Fig. $5 \mathbf{b}$, curve 3 quite satisfactorily coincides with the dependence $\Theta_{D}(T)$ of the FCC crystal (curve 2). This is manifested in the behavior of $\Theta_{D}(T)$ at $T \leq 0.1 \Theta_{P}$ and in the coincidence of the minima (both in temperature and in magnitude). Thus, one can assert that the dependence $\Theta_{D}(T)$ at low temperatures is conditioned by the changes in the character of the phonon propagation on the frequency of the first van Hove singularity.

Taking into account the Einstein level tailing can improve the approximation of the $\Theta_{D}(T)$ function at low temperatures (curves 4).

As mentioned above, the frequency of the first van Hove singularity $\omega$ * is an "interface" frequency between the fast and slow phonons, i.e. between propagons and diffusons. It can be interpreted as the Ioffe-Regel singularity (or its equivalent) in a regular crystal system.

Maxima on the ratio $v(\omega) / \omega^{2}$ can be considered as BPs only when $\omega<\omega^{*}$, because the maximum on the mentioned ratio, corresponding to the first van Hove singularity, always exists. Within this frequency interval the phonon density can be approximated by a parabola, and its deviation from the Debye density $v_{D}^{(3)}(\omega)$ can be expressed by the frequency dependence of the value $\omega_{D}$, i.e. writing the phonon density in a form analogous to (9). At $q=3$ we have

$$
v(\omega) \equiv \frac{3 \omega^{2}}{\omega_{D}^{3}(\omega)} .
$$




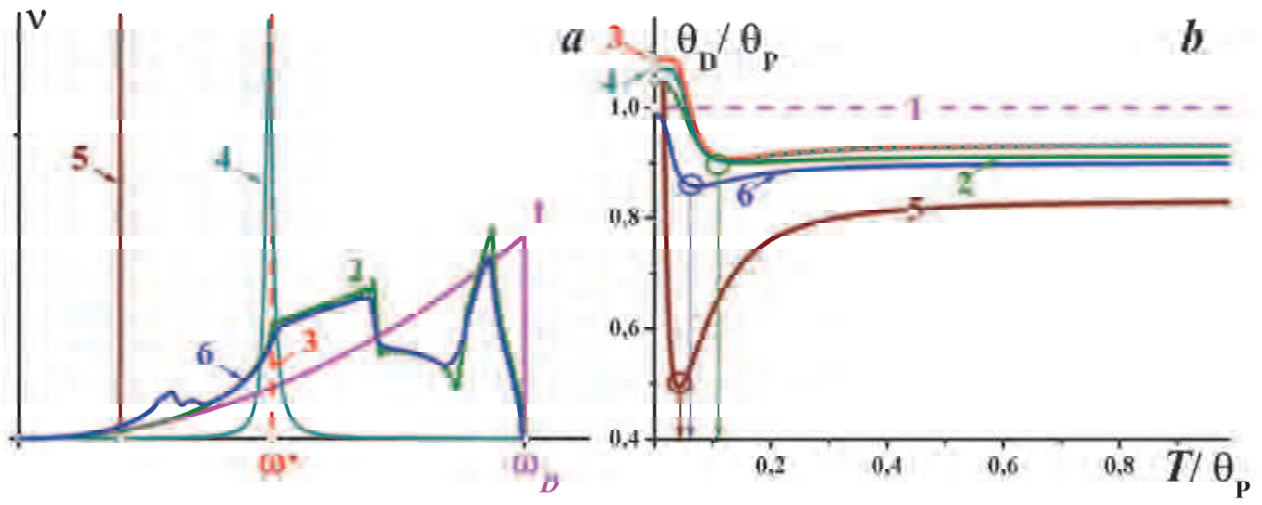

Fig. 5. Relationship of the temperature dependence of the Debye temperature (part $\mathbf{b}$ ) to the character of the long-wavelength phonons propagation in a crystal (part a).

Then, using the definition of $\omega_{D}$, the ratio of the phonon density to the squared frequency can be expressed by the dispersion of sound velocities $s_{i}(\omega)$

$$
\frac{v(\omega)}{\omega^{2}} \equiv \frac{3}{\omega_{D}^{3}(\omega)}=\frac{V_{0}}{6 \pi^{2}} \sum_{i=1}^{3} \frac{1}{s_{i}^{3}(\omega)},
$$

where $V_{0}$ is the unit cell volume. Thus, the occurrence of the maximum on the ratio $v(\omega) / \omega^{2}$ is caused by the additional dispersion of sound velocities. This dispersion is caused by the heterogeneity of the structure, which is the source of quasi-localized vibrations. Such additional sound velocity dispersion must be manifested in the behavior of the temperature dependence $\Theta_{D}$. On the curve $\Theta_{D}(T)$ a low-temperature minimum should appear (see curve 5, Fig. 5b), deeper than those on curves 1-4 in Fig. 5b. This curve corresponds, in addition to the quasi-localized perturbations on the frequency of the first van Hove singularity in the phonon spectrum with the density of states $v_{\text {appr }}(\omega)$, to the presence of an additional resonance level with the frequency $\delta\left(\omega-\omega_{D} / 5\right)$ (see Fig. $5 \mathbf{a}$ ). Curves 6 in both parts of Fig. 5 correspond to the 5\% solution of a heavy isotope impurity in the FCC crystal. The formation of the QLV leads to a significant deepening of the $\Theta_{D}(T)$ low-temperature minimum and to be shifting of its temperature below that of the perfect crystal.

In the first section it was shown that heavy or weakly bound impurities form QLV caused by their motion. On these vibrations the fast acoustic phonons associated with the displacements of atoms of the host lattice are scattered. This leads to kinks in the contribution to the phonon spectral density (see curve 6 in Fig. 3) which are a manifestation of the Ioffe-Regel crossover. On the background of large quasi-local maxima it is difficult to distinguish their influence on the vibrational characteristics of the crystal. The study of this effect is possible in systems in which interatomic interactions are not accompanied by the formation of QLV, or in systems in which the frequencies of QLV lie beyond the propagon zone. Examples of such systems are crystals with weakly bound impurities. Fig. 6 shows the low-frequency parts of the phonon density of states $(a)$ and the temperature dependence $\Theta_{D}(b)$ for the FCC lattice, in which force constants of impurities $(p=5 \%)$ are four and eight 
times weakened (curves 3 and 4 , respectively). Part $a$ shows the functions $\frac{v(\omega)}{4}\left(\frac{\omega_{m}}{\omega}\right)^{2}$ (curves $3^{\prime}$ and $4^{\prime}$ ), for which the deviation of the phonon density of states from the Debye form is more pronounced. Curve 1 corresponds to a perfect crystal. Curve 2 shows the frequency dependence of the group velocity in the direction $\Gamma L$ (see Fig. 1a). Values $\omega_{q l}^{(4)}$ and $\omega_{q l}^{(8)}$ correspond to the frequencies of QLV in a lattice containing an isolated weakly bound impurity $\left(\alpha^{\prime} / \alpha=1 / 4\right.$ and $\alpha^{\prime} / \alpha=1 / 8$, respectively). As can be seen from the figure, the phonon densities are qualitatively different from the quasi-Debye behavior, starting from the frequencies $\omega=\omega_{q l}^{(4)}$ (curve 3) and $\omega=\omega_{q l}^{(8)}$ (curve 4). In this system the formation of QLV with such frequencies corresponds to the existence of atoms with few weakened force interactions (at least two, along the same line), i.e. to the formation of defect clusters (or impurity molecules). The minimum size of the defect cluster is equal to two interatomic distances and the Ioffe-Regel crossover can occur in a wide range of values (see Fig. $1 b$ ). Fig. $6 \boldsymbol{b}$ shows that there are notable low-temperature minima on $\Theta_{D}(T)$ for crystals with impurities $(p=5 \%)$ whose force interactions are four and eight times weakened (curves 3 and 4, respectively). These minima points to a slowdown of acoustic phonons due to their localization on the defect clusters and due to the scattering of additional phonons, remaining delocalized on the resulting quasi-localized states.

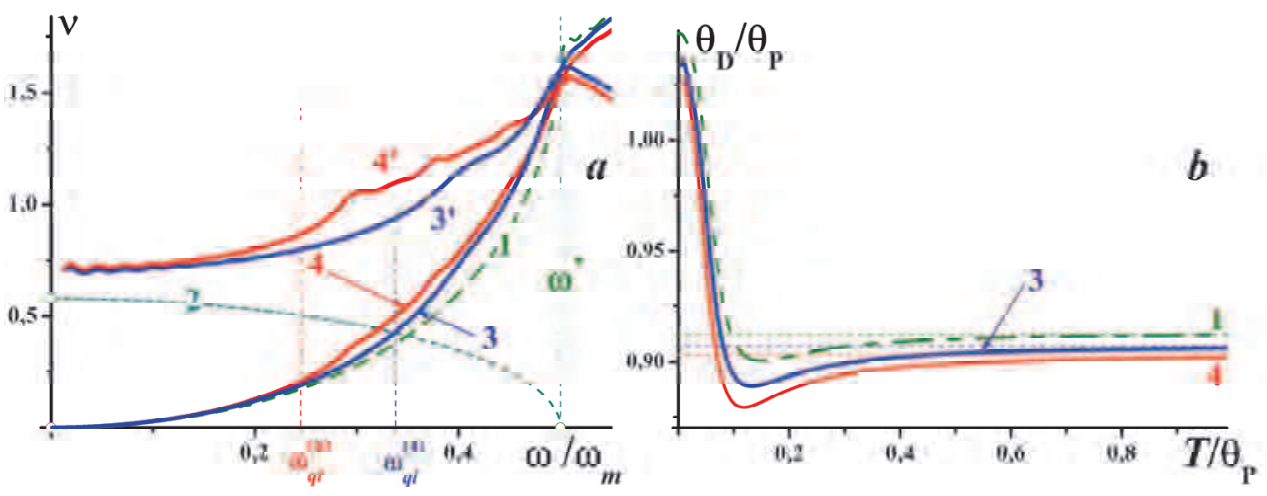

Fig. 6. Low-frequency parts of phonon spectra (part a) and temperature dependences $\Theta_{D}$ (part b) of FCC crystals with 5\% of weakened force interactions

The high sensitivity of the low-temperature heat capacity to the slowing of the longwavelength phonons is clearly manifested in the case when not only the interaction of impurity atoms with the host lattice is weakened, but also the interaction between substitution impurities in the matrix of the host lattice. An example of such a system is the solid solution $\mathrm{Kr}_{1-\mathrm{p}} \mathrm{Ar}_{\mathrm{p}}$. Krypton and argon are highly soluble in each other and the concentration $\mathrm{p}$ can take any value from zero to one. Argon is $\approx 2.09$ times lighter than krypton, and the interaction of the impurity of argon with krypton atoms is slightly weaker than the interaction of krypton atoms between each other, so an isolated Ar impurity in the 
$\mathrm{Kr}$ matrix behaves almost like a light isotope. At the same time, in a krypton matrix the interaction of argon impurities between each other is more than five times weaker than the interaction between the krypton atoms (Bagatskii et al., 2007). Fig. 7a shows the phonon densities of states of pure krypton and argon as well as that of the $\mathrm{Kr}_{0.756} \mathrm{Ar}_{0.244}$ solid solution. At such a concentration there is a sufficient number of isolated impurities and defect clusters with dimensions less than two interatomic distances in the solution (Fig. 7b). This leads, in comparison with the pure $\mathrm{Kr}$ phonon spectrum, to the increase of the number of high-frequency states in the phonon spectrum of the solution (Bagatskii et al., 1992). In such clusters weakly coupled argon impurities are not created and quasi-local vibrations are not formed. At the same time in such a solution larger defect clusters are formed, which consists of weakly coupled Ar impurities. However, the frequency of QLV formed by these clusters is $\omega_{q l}^{\mathrm{Kr}-\mathrm{Ar}} \approx 0.86 \omega_{\mathrm{Kr}}^{*}$, that is (unlike the previous case) slightly less than the frequency of the first van Hove singularity for the Kr lattice. Therefore, neither on the solution phonon density of states nor on its relationship to the square frequency any singularities do appear. Extension of the of quasi-continuous spectrum of the Kr-Ar solution as compared with pure $\mathrm{Kr}$, as seen in Fig. 7a, occurs mainly due to the phonons with frequencies in the interval $\left[\omega^{*}, \omega^{* *}\right]$ (diffuson zone).

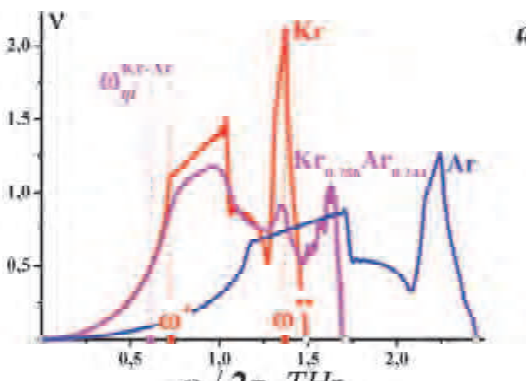

क $/ 2 \pi, T H z$

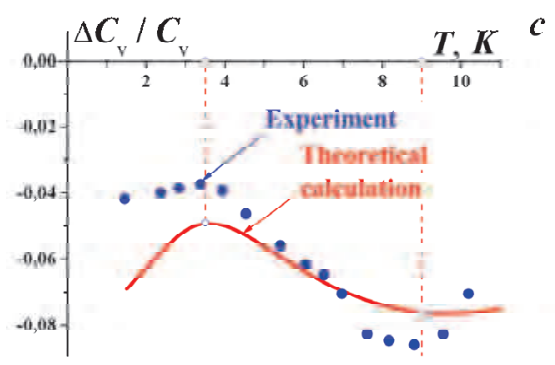

$a$
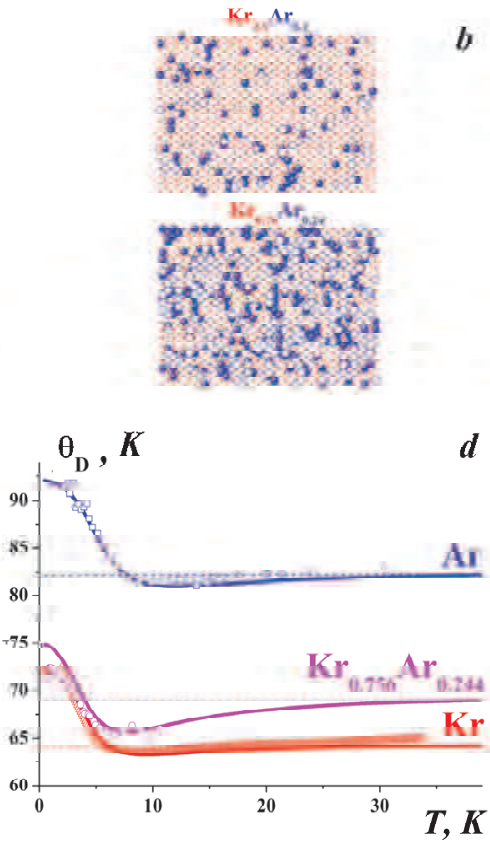

Fig. 7. Phonon densities (a) and temperature dependences of the Debye temperature (d) of the krypton, argon and the $\mathrm{Kr}_{0.756} \mathrm{Ar}_{0.244}$ solid solution. Part $\mathbf{b}$ shows in the [111] plane, some typical configurations of the displacements of argon impurity in the in krypton matrix at $p=0.1$ and at 0.24 (circles and filled circles correspond to the Ar atoms, lying in different neighboring layers). Part $\mathrm{c}$ is shows the relative change of the heat capacity. 
Note that the phonon densities of states of the solution and of pure krypton are practically the same in the most part of the propagon zone. The redistribution of the phonon frequency leads to a characteristic two-extremum behavior of the temperature dependence of the relative change of the low-temperature heat capacity (Fig. 7c), the maximum on which indicates that there is an additional slowing-down of the long-wavelength acoustic phonons on slow phonons, corresponding to the quasi-local vibrations of weakly couple argon atoms. This scattering, as in earlier cases, forms a significant minimum in the temperature dependence of $\Theta_{D}$. Fig. 7d plots the values $\Theta_{D}(T)$ for pure krypton, argon, and the $\mathrm{Kr}_{0.756} \mathrm{Ar}_{0.244}$ solution. These dependences are the solutions of the transcendental equation (10) for the heat capacity, calculated theoretically and determined experimentally, see Fig. 7c (Bagatskii et al., 1992). The results of the theoretical calculations show a good agreement with experimentally obtained results, especially near the minimum on $\Theta_{D}(T)$. This minimum can appear also in the case when the maximum of the ratio $v(\omega) / \omega^{2}$ is not observed.

Thus, the results presented in this section allow us to make the conclusion that both the low temperature heat capacity and the temperature dependence of the value $\Theta_{D}$ are highly sensitive not only to the formation of quasi-localized states, but also to the reduction of the rate of propagation of long-wavelength acoustic phonons due to their scattering on these states. This slowdown is clearly manifested in the frequency range as boson peaks in the ratio $v(\omega) / \omega^{2}$, or as another singularities of the Ioffe-Regel type, but only when certain conditions are fulfilled. They are, according to our analysis:

1. For such defects as local weakening of the interatomic interactions or light weakly bound impurities the QLV scattering frequency must be low enough, and so, in other words, the "power of the defect" should be large enough.

2. Defect cluster should be large enough (at least two atomic distances) which requires a high enough $(\sim 15-20 \%)$ concentration of defects.

\section{Low-frequency features of the phonon spectra of layered crystals with complex lattice}

As it has been shown in the previous sections the low-frequency region of the phonon density of states of heterogeneous systems differs from the Debye form. This is caused by the formation of the quasi-localized states on the structure heterogeneities and by the scattering of the fast longwavelength acoustic phonons (propagons) on them. However, it is not necessary that these heterogeneities were defects violating the regularity of the crystalline arrangement of atoms. If, in the crystal with polyatomic unit cell the force interaction between atoms of one unit cell is much weaker than the interaction between cells, then optical branches occur in the phonon spectrum of the crystal at the frequencies significantly lower than the compound Debye frequency. These optical branches are inherent to the phonon spectra of many highly anisotropic layered crystals and they may cross the acoustic branches, causing additional features in the propagon area of phonon spectrum (Wakabayashi et al., 1974; Moncton et al., 1975; Syrkin \& Feodosyev, 1982). Note that the deviation of the phonon spectrum of such compounds from $v_{D}^{(3)}(\omega)$ at low frequencies may be a manifestation of their quasi-low-dimensional structure as well 
(Tarasov, 1950) of the flexure stiffness of single layers (Lifshitz, 1952b). However, the crossing of the low-lying optical modes with the acoustic ones may also occur in systems, in whose propagon zone of the phonon spectrum no quasi-low-dimensional peculiarities and no flexural vibrations are present. These compounds include high-temperature superconductors, dichalcogenides of transition metals, a number of polymers and biopolymers, as well as many other natural and synthesized materials. A distinctive feature of the structure of these substances is the alternation of layers with strong interatomic interactions (covalent or metal) with layers in which atomic interactions are much weaker, e.g. the van der Waals interaction. Since this interaction is weak along all directions, the propagation of the propagons is three-dimensional and can be characterized by the temperature dependence of the $\Theta_{D}$ determined by formulas $(10,11)$.

Let us examine a simple model of such a structure, i.e. the system based on a FCC crystal lattice and generated by "separating" the atomic layers along the [111] axis into a structure consisting of stacked layers of the closely packed ...A - B - B - A - B - B - ... type. To describe the interatomic interaction we shall restrict our attention to the central interaction between nearest neighbors. We assume that the interaction between atoms of the B type (lying in one layer as well as in different layers) is half as strong as the interaction between A type atoms and atoms of different types (we assume these interactions are the same). The phonon spectrum of considered model contains nine branches (three acoustic and six optical) and the optical modes are not separated from the acoustic modes by a gap. The frequencies of all phonons polarized along the [111] axis (axis $c$ ) lie in the low-frequency region. At $k=0$ two optical modes have low frequencies corresponding to a change in the topology of the isofrequency surfaces (from closed one to the open one along the $c$ axis) both for transverse and longitudinal modes. Thus, these frequencies play the role of the van Hove frequencies $\omega^{*}$ and are shown in Figs. 8a-d and 9a as vertical dashed lines $\omega_{\tau}^{*}$ and $\omega_{l}^{*}$.

Fig. 8 displays the spectral densities corresponding to displacements of $\mathrm{A}$ and $\mathrm{B}$ atoms in the basal plane $a b$ and along the $c$ axis (curves 1). The normalization of each spectral density corresponds to its contribution to the total phonon density of states $v(\omega)$ presented in Fig. 9a:

$$
v(\omega)=\frac{2}{9} \cdot \rho_{a b}^{(\mathrm{A})}(\omega)+\frac{1}{9} \cdot \rho_{c}^{(\mathrm{A})}(\omega)+\frac{4}{9} \cdot \rho_{a b}^{(\mathrm{B})}(\omega)+\frac{2}{9} \cdot \rho_{c}^{(\mathrm{B})}(\omega) .
$$

Fig. 8 also displays the quantities proportional to the ratio of the corresponding spectral densities to the squared frequency (curves 2). The coefficients of proportionality are chosen so that these curves may be placed in the same coordinate system as the corresponding spectral density. The functions $\rho_{c}^{(\mathrm{A})}(\omega)$ and $\rho_{c}^{(\mathrm{B})}(\omega)$ and their ratios to $\omega^{2}$ have distinct features at $\omega_{l}^{*}$ as well as at a certain frequency $\omega_{c}$ lying below $\omega_{\tau}^{*}$. This frequency corresponds to the crossing of the longitudinal acoustic mode, polarized along the $c$ axis, with the transversely polarized optical mode propagating in the plane of the layer. The velocity of sound in this acoustic mode is $s_{l}^{(c)} \sim \sqrt{C_{33}}$ (in the described model the elastic moduli of elasticity $C_{i k}$ satisfy the relations $\left.C_{11}=2.125 \cdot C_{33}=3 \cdot C_{66}=7.5 \cdot C_{44}\right)$. The spectral densities $\rho_{a b}^{(\mathrm{A})}(\omega)$ and $\rho_{a b}^{(\mathrm{B})}(\omega)$ have additional features at frequencies $\omega_{a b}^{(\tau)}<\omega^{*}$ and $\omega_{a b}^{(l)} \in\left[\omega_{\tau}^{*}, \omega_{l}^{*}\right]$. These features are related to the crossing of acoustic branches with the low- 
frequency optical mode which is polarized along the $c$ axis. There are three acoustic waves propagating in the basal plane and differing substantially from one another (longitudinal wave $s_{l}^{(a b)} \sim \sqrt{C_{11}}$ and two transverse waves). One of the transverse waves is polarized in the basal plane $\left(s_{\tau}^{(a b)} \sim \sqrt{C_{66}}\right)$ and another one is polarized along the $c$ axis $\left(s_{n}^{(a b)} \sim \sqrt{C_{44}}\right)$. The acoustic modes with sound velocities $s_{l}^{(a b)}$ and $s_{\tau}^{(a b)}$ cross the low-frequency optical mode. In this optical mode at $k=0$ the frequency of the vibrations is $\omega=\omega_{l}^{*}$, and at the point $\mathrm{K}$ at the boundary of the first Brillouin zone (see Fig 1) the mode joins the slowest acoustic mode, polarized along the $c$ axis. Appreciable dispersion of this optical mode leads to a small value of $\omega_{a b}^{(\tau)}\left(\omega_{a b}^{(\tau)}<\omega_{c}\right)$ and to the blurring of the feature near $\omega_{a b}^{(l)}$.
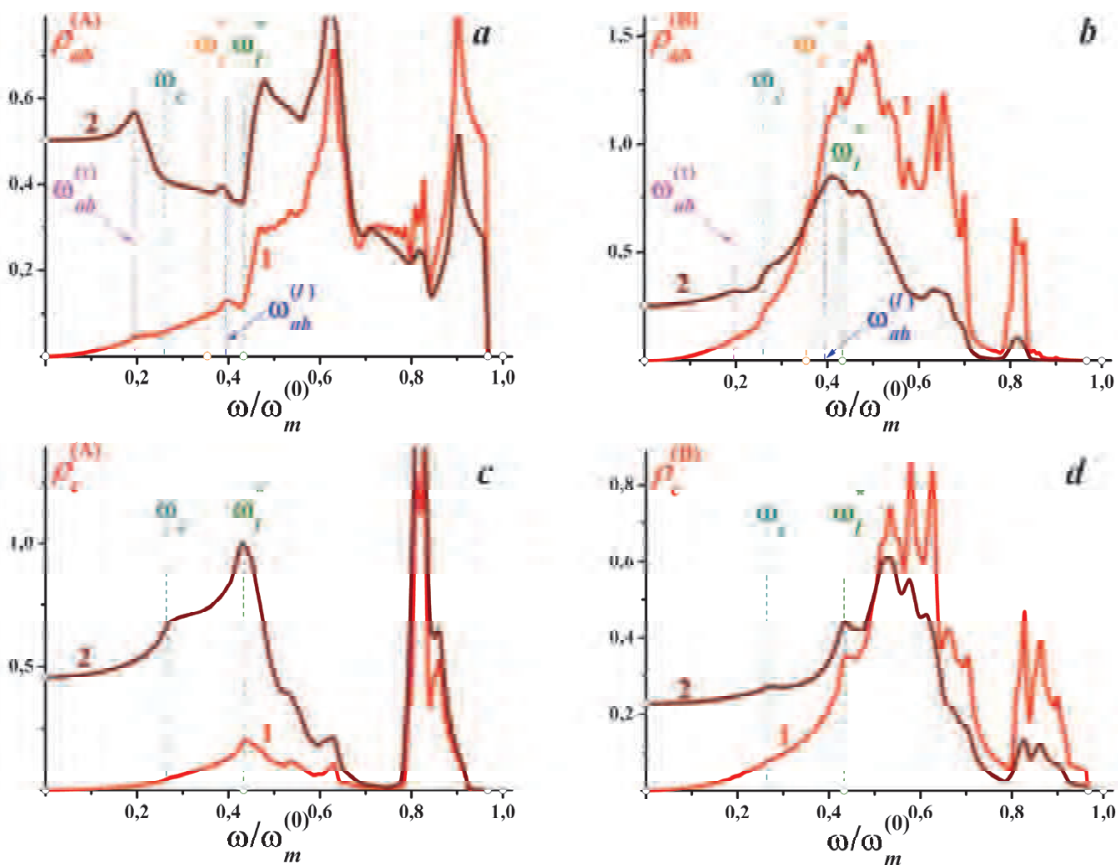

Fig. 8. Spectral densities (curves 1) and their ratio to the squared frequency (curves 2), corresponding to displacements of atoms of different sublattices along different crystallographic directions.

All spectral densities at quite low frequencies are proportional to $\omega^{2}$, i.e. at lowtemperatures the thermodynamic quantities should be determined by an ordinary threedimensional behavior (see Fig. 8). Fig. $9 \mathbf{b}$ shows the temperature dependence of the Debye temperature $(10,11)$ for the considered model. For comparison, on Fig. $9 \mathbf{a}$ and $9 \mathbf{b}$ the characteristics of the "initial" FCC lattice is shown (lattice of $A$ type atoms). As a result of the weakening (as compared to the $A$ lattice) of some force bonds the function $v(\omega)$ increases at low frequencies (Fig. 9a) and therefore $\Theta_{D}$ decreases. The scattering of the propagons on slow optical phonons forms a distinct low-temperature minimum on $\Theta_{D}(T)$. 

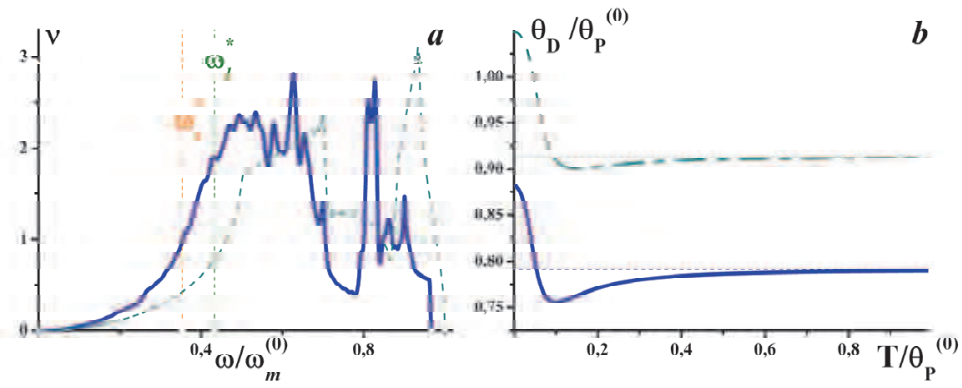

Fig. 9. Phonon density of states (a) and temperature dependence of $\Theta_{D}(T)$ (b) of a layered crystal with a three-atom unit cell (solid curves) and analogous characteristics of an ideal FCC lattice with central interaction of the nearest neighbors (dashed curves).

The Ioffe-Regel crossover determined by the intersections of the acoustic branches with the low-lying optical one is clearly apparent on the niobium diselenide phonon spectrum. This compound has a three-layer Se-Nb-Se "sandwich" structure. Fig. 10 (center) shows the dispersion curves of the $\mathrm{NbSe}_{2}$ low-frequency branches (Wakabayashi et al., 1974)]. The lowfrequency optical modes $\Delta_{2}$ and $\Delta_{5}$ correspond to a weak van der Waals interaction between "sandwiches". They cross at points C2, C3, C4, S1, A1 and A2 with acoustic branches polarized in the plane of layers. The wavelength $\lambda_{\text {eff }}$ (see Sec. 2) corresponding to frequency of each of these crossovers exceeds the thickness $h$ of the "sandwich". The parameter $h$ plays in this case the same role as the distance between impurities in solid solutions, i.e. the condition of the Ioffe-Regel is met. Therefore, for given values of frequency as well as for the van Hove frequencies (points D1, D2 and D4) an abrupt change of the propagon group velocity occurs. This leads to the appearance of peaks on the dependences $v(\omega)$ and $v(\omega) / \omega^{2}$ (curves 1 and 2 in Fig. 10a) and to the formation of a rather deep lowtemperature minimum in the dependence $\Theta_{D}(T)$ (Fig. 10b). For the longitudinal acoustic mode $\Delta_{1}$ polarized along the $c$ axis at the frequency corresponding to the point of its intersection with the branch $\Delta_{5}$ (point C1), the value $\lambda_{\text {eff }}$ is less than $h$. Therefore, at this point the group velocity of phonons does not have a jump and does not change its sign. There are no peculiarities at point $\mathrm{C} 1$ on the phonon density of states and on the function $v(\omega) / \omega^{2}$.

Thus, in the crystalline ordered heterogeneous structures the scattering of fast phonons on slow optical ones is possible. This scattering is similar to the scattering of such phonons on quasi-localized vibrations in disordered systems and is completely analogous to that considered in (Klinger \& Kosevich, 2001, 2002). It leads to the formation of the same lowfrequency peculiarities on the phonon density of states than are those manifested in the behavior of low-temperature vibrational characteristics. The elastic properties of structures discussed in this section differ essentially from the properties of low-dimensional structure. However, at high frequencies (larger than the frequencies of the van Hove singularities, which correspond to the transition from closed to open isofrequency surfaces along the $c$ axis) the phonon density of states exhibits quasi-two dimensional behavior seen on parts $a$ of Figs. 8, 9 and 10. Such a behavior is inherent to many heterogeneous crystals, in particular high-temperature superconductors (see, e. g., Feodosiev et al., 1995; Gospodarev et al., 1996), 
as was confirmed experimentally (Eremenko et al., 2006). This allows us to describe the vibrational characteristics of such complex compounds in the frames of low-dimensional models.
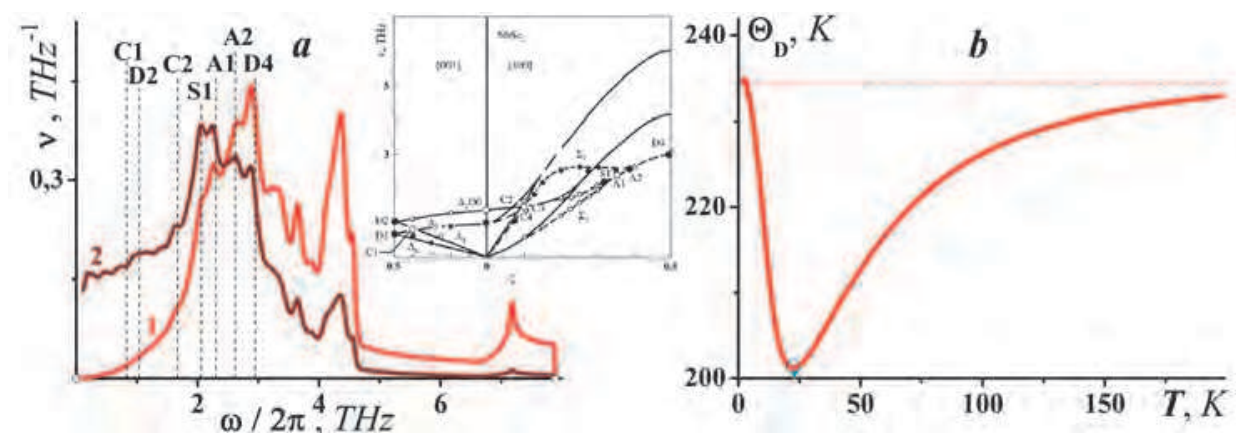

Fig. 10. Vibrational characteristics of $\mathrm{NbSe}_{2}$. Part a shows the phonon density of states (curve 1) and ratio $v(\omega) / \omega^{2}$ (curve 2). On the inset the dispersion curves of the lowfrequency vibration modes determined by the method of neutron diffraction are shown. Part $\mathbf{b}$ shows the dependence $\Theta_{D}(T)$.

The theory developed for the multichannel resonance transport of phonons across the interface between two media (Kosevich Yu. et al., 2008) can be applied to interpret the experimental measurements of the phonon ballistic transport in an Si-Cu point contact (Shkorbatov et al., 1996, 1998). These works revealed for the first time the low temperature quantum ballistic transport of phonons in the temperature region $0.1-3 \mathrm{~K}$. Besides, in some works (Shkorbatov et al., 1996, 1998) a reduced point contact heat flux in the regime of the geometric optics was investigated in the temperature interval $3-10 \mathrm{~K}$. The results obtained in these works showed that in this temperature interval the reduced heat flow through the point contact is a non-monotonous temperature function and has pronounced peaks at temperatures $\mathrm{T}_{1}=4.46 \mathrm{~K}, \mathrm{~T}_{2}=6.53 \mathrm{~K}$ and $\mathrm{T}_{3}=8.77 \mathrm{~K}$. We suppose that the series of peaks for the reduced heat flow (Shkorbatov et al., 1996, 1998) could be explained by the models represented in Fig.11 a,b. These peaks are a result of the resonance transport. In the case of the single-channel resonance transport studied in work (Feher et al., 1992) a model of the narrow resonance peak was applied, meaning the following: the total heat flux $\dot{Q}$ may be written as the sum of the ballistic flux $\dot{Q}_{B}$ and the resonance heat fluxes $\dot{Q}_{R}, \dot{Q}=\dot{Q}_{B}+\dot{Q}_{R}$. Assuming the narrow resonance peak near the frequency $\omega_{0}^{\alpha}$ we obtain the formula describing the temperature dependence of the heat flux:

$$
\dot{Q}\left(T, T_{0}\right)=C \Delta T^{4}+\sum_{\alpha} K_{\alpha}\left[\frac{1}{\exp \left(\hbar \omega_{0}^{\alpha} / T\right)-1}-\frac{1}{\exp \left(\hbar \omega_{0}^{\alpha} / T_{0}\right)-1}\right] .
$$

To separate the two parts of the total heat flux, its value must be divided by $\Delta T^{4}=\left(T^{4}-T_{0}^{4}\right)$.

This model (using only one frequency) can be fitted to our experimental data with a correlation factor of about 0.95 . The resonance frequency $\omega_{0}$ is connected with $T_{\max }$ by the 

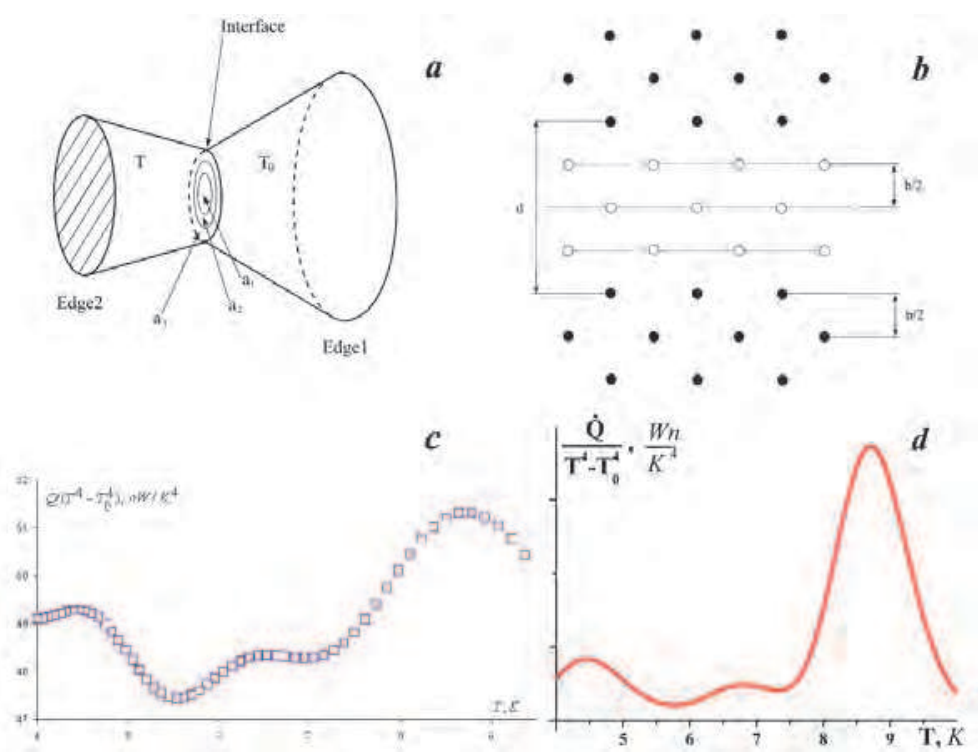

Fig. 11. a) Schematic model of a contact. $\mathbf{T}$ and $\mathbf{T}_{\mathbf{0}}$ are the temperatures of the massive edges of the contact; $\mathbf{a}_{1}, \mathbf{a}_{2}$, and $\mathbf{a}_{3}$ are the zones with different composition of the interface layer. b) Schematic figure showing an interface between two crystal lattices that contains three intercalate impurity layers. c) Experimentally observed temperature dependence of the reduced heat flux through the $\mathrm{Si}-\mathrm{Cu}$ point contact. d) Results of a numerical calculation using the considered model.

relation $\hbar \omega_{0}=3.89 T_{\max }$. Using the model of the multichannel resonance transport we modified the expression (15) in a following way:

$$
\frac{\dot{Q}}{\Delta T^{4}}=\frac{1}{\Delta T^{4}} \sum_{n=1}^{3} K_{n}\left[\left(\exp \left(3.89 \frac{T_{n}}{T}\left(1+\frac{1}{T_{s}^{2}}\left(T-T_{n}\right)^{2}\right)\right)-1\right)^{-1}-\left(\exp \left(3.89 \cdot \frac{T_{n}}{T_{0}}\right)-1\right)^{-1}\right]+C
$$

The optimal correspondence between the values calculated by this formula and the experimental results was obtained for the following values of parameters:

$$
\begin{aligned}
& T_{0}=0.15 \mathrm{~K} ; T_{1}=4.46 \mathrm{~K} ; T_{2}=6.8 \mathrm{~K} ; T_{3}=8.71 \mathrm{~K} ; T_{\mathrm{s}}=1.5 \mathrm{~K} . \\
& K_{1}=0.7 \mathrm{nW} ; K_{2}=2 \mathrm{nW} ; K_{3}=50 \mathrm{nW} ; C=49.55 \mathrm{nW} / \mathrm{K}^{4} .
\end{aligned}
$$

The expression (16) takes into account the presence of three channels of the resonance transport as well as (using an additional term containing the intrinsic temperature $T_{S}$ ) the instability of the intermediate layer of weakly bound impurities near the resonance. Results of numerical calculations by formula (16) are given in Fig.11d. These results evidence that the proposed model describes in much detail the experimental results presented in Fig. 11c. It should be noted that the temperature $T_{S}$ used in our calculations corresponds to the binding energy of the impurity layer with contact banks. This temperature is by two orders of magnitude lower than the Debye temperature of crystals forming the banks of contacts. 


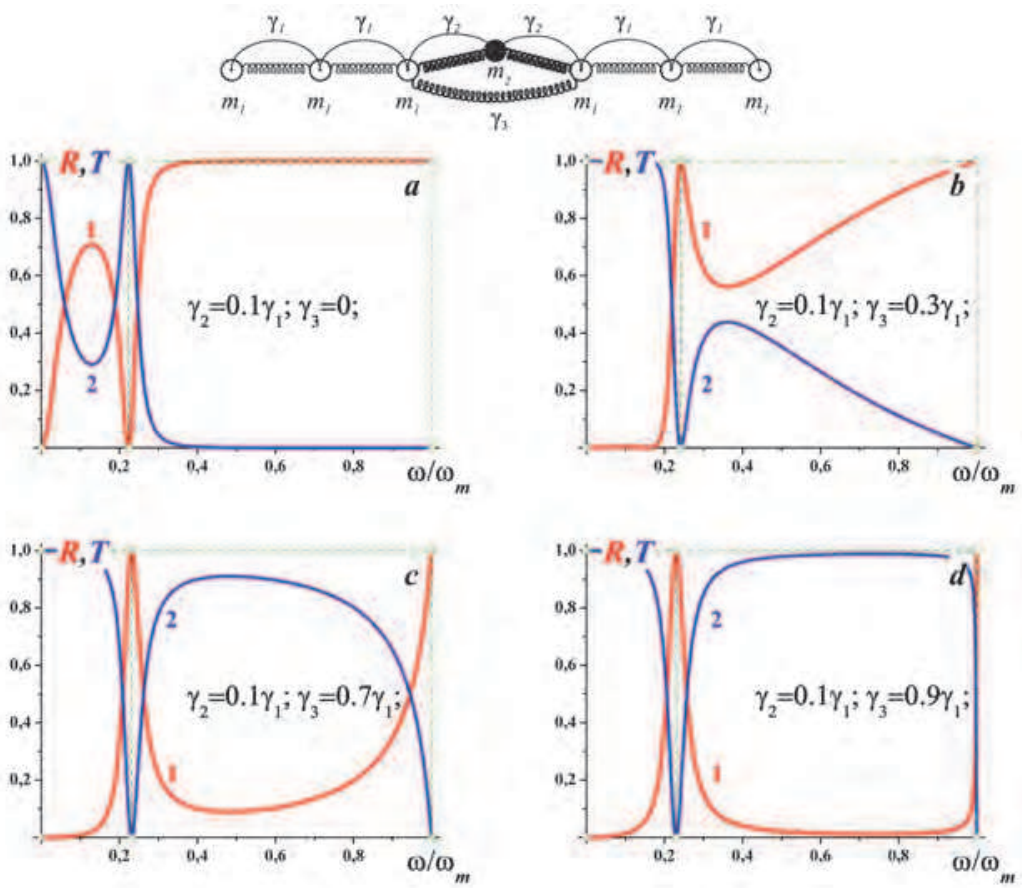

Fig. 12. Coefficients of the phonon energy reflection (curve 1, red line) and transmission (curve 2, blue line) through an impurity atom.

This is in agreement with the fact that the binding constant of the impurity layer with contact banks is by two orders of magnitude lower than the binding constant in crystals forming this contact (Shklyarevskii et al., 1975; Koestler et al., 1986; Lang, 1986). Coefficients $K$ are proportional to the squares of the area of different interface layers. Using the results presented in Fig. 11d we can interpret experimental results (Shkorbatov et al., 1996, 1998) presented in Fig. 11c.

Finally we consider the resonance reflection and transmission of phonons through an intercalated layer between two semi-infinite crystal lattices. We consider an infinitely long chain which contains a substitution impurity atom weakly coupled to the matrix atoms (see model in Fig. 12). In this system quasi-local (resonance) impurity oscillations emerge with such a frequency, at which the transmission coefficient through the impurity becomes equal to unity (full phonon transmission through the interface, see Fig.12a). Let us compare these results with the results received taking into account the force constant $\gamma_{3}$, corresponding to the interaction between non-nearest neighbors. We have shown that if the non-nearest neighbor force constant $\gamma_{3}$ is larger than the weak bounding force constant $\gamma_{2}$ (Kosevich, et al., 2008) (see Fig.12), two frequency regions with enhanced phonon transmission are formed, separated by the frequency region with enhanced phonon reflection. Namely, for $\gamma_{3} \approx \gamma_{1}$ a strong transmission "valley" occurs at the same resonance frequency at which there is a transmission maximum for $\gamma_{3}<\gamma_{2}<\gamma_{1}$. Moreover, this transmission minimum occurs on the background of an almost total phonon transmission through the impurity atom due to the strong interaction of matrix atoms through the defect (with force constant $r_{3} \approx r_{1}$ ). For 
large values of $\gamma_{3}$, the resonant transmission frequency corresponds to the frequency of total reflection (so-called Fano effect) (Fano, 1961). Such a system permits to make a filter which reflects the phonons in a very narrow region of frequencies (heat transmission is minimum at corresponding temperatures) while the total transmission is observed in other regions of frequencies. It is worth to mention that such an inversion of the transmission and reflection spectra in the two limiting cases is directly related to the Fano-type interference. Similar inversion of the Fano-type transmission and reflection resonances also occurs in sound transmission through two-dimensional periodic arrays of thin-walled hollow cylinders due to the their flexural vibration modes (see Liu, 2000).

\section{Acknowledgments}

This work was supported by the grants of the Ukrainian Academy of Sciences under the contract No. 4/10-H and by the grant of the Scientific Grant Agency of the Ministry of Education of Slovak Republic and the Slovak Academy of Sciences under No. 1/0159/09.

\section{References}

Ahmad N., Hutt K.W. \& Phillips W.A. (1986) Low-frequency vibrational states in $\mathrm{As}_{2} \mathrm{~S}_{3}$ glasses. J. Phys. C.: Solid State Phys. , Vol.19, pp. 3765 - 3773.

Allen P.B., Feldman J.L., Fabian J. \& Wooten F. (1999) Diffusons, locons and propagons: character of atomic vibrations in amorphous Si. Philosophical Magazine B. Vol. 79, pp. 1715-1731.

Arai M., Inamura Y. \& Otomo T. (1999) Novel dynamics of vitreous silica and metallic glass. Philosophical Magazine B. Vol. 79, pp. 1733 - 1739.

Bagatskii M.I., Syrkin E.S. \& Feodosyev S.B. (1992) Influence of the light impurities on the lattice dynamics of cryocrystals. Low Temp. Phys. Vol. 18, pp. 629-635.

Bagatskii M.I., Feodosyev S.B., Gospodarev I.A., Kotlyar O.V., Manzhelii E.V. Nedzvetskiy A.V. \& Syrkin E.S. (2007) To the theory of rare gas alloys: heat capacity. Low Temp. Phys. Vol. 33, pp. 741-746.

Buchenau U., Nücker N. \& Dianoux A. J. (1984) Neutron Scattering Study of the LowFrequency Vibrations in Vitreous Silica. Phys. Rev. Lett., Vol. 53, pp. 2316 - 2319

Buchenau U., Wisschnewski A., Ohl M. \& Fabiani E. (2007) Neutron scattering evidence on the nature of the boson peak. J. Phys.: Condens. Matter Vol. 19, pp. 205106.

Cape J.A., Lehman G.M., Johnston W.V. \& de Wames R.E. (1966) Calorimetric observation of virtual bound-mode phonon states in dilute $\mathrm{Mg}-\mathrm{Pb}$ and $\mathrm{Mg}-\mathrm{Cd}$ alloys. Phys. Rev. Lett. Vol. 16, pp. 892 - 895.

Duval E., Saviot L., David L., Etienne S. \& Jalbibitem J.F. (2003) Effect of physical aging on the low-frequency vibrational density of states of a glassy polymer. Europhys. Lett. , Vol. 63, pp. 778 - 784.

Eremenko V.V., Gospodarev I.A., Ibulaev V.V., Sirenko V.A., Feodosyev S.B. \& Shvedun M.Yu. (2006) Anisotropy of $\mathrm{Eu}_{1+x}\left(\mathrm{Ba}_{1-\mathrm{y}} \mathrm{Ry}\right)_{2-\mathrm{x}} \mathrm{Cu}_{3} \mathrm{O}_{7-\mathrm{d}}$ lattice parameters variation with temperature in quasi-harmonic limit. Low Temp. Phys. Vol. 32, pp. 1560-1565.

Fano U. (1961) Effects of Configuration Interaction on Intensities and Phase Shifts. Phys. Rev. Vol. 124, pp. 1866 - 1878.

Feher A., Stefanyi P., Zaboi R., Shkorbatov A.G. \& Sarkisyants T.Z. (1992) Heat conductivity of dielectric- dielectric and metal- dielectric point contacts at low temperatures. Sov. J. Low Temp. Phys. Vol. 18, pp. 373 - 375. 
Feher A., Yurkin I.M., Deich L.I., Orendach M. \& Turyanitsa I.D. (1994) The comparative analysis of some low-frequency vibrational state density models of the amorphous materials - applied to the $\mathrm{As}_{2} \mathrm{~S}_{3}$ glass. Physica B, Vol. 194 - 196, pp. 395 - 396.

Fedotov V.K., Kolesnikov A.I., Kulikov V.I., Ponjtovskii V.G., Natkanec I., Mayier Ya. \& Kravchik Ya. (1993) The studing of vibration anharmonisms of copper and oxygen atoms in the yttrium ceramics by inelastic neutron scattering. Fiz. Tv. Tela., Vol. 35, pp. 310-319 (in Russian).

Feodosyev S.B., Gospodarev I.A. Kosevich A.M. \& Syrkin E.S. (1995) Quasi-LowDimensional Effects in Vibrational Characteristics of 3D-Crystals. Phys. Low-Dim. Struct. , Vol. 10-11, pp. 209 - 219

Gospodarev I.A., Kosevich A.M., Syrkin E.S. \& Feodosyev S.B. (1996) Localized and quasilow-dimensional phonons in multilayered crystals of HTS type. Low Temp. Phys. Vol. 22, pp. 593 - 596

Gospodarev I. A., Grishaev V. I., Kotlyar O. V., Kravchenko K. V., Manzheliǔ E. V., Syrkin E. S. \& Feodosyev S. B. (2008) Ioffe_Regel crossover and boson peaks in disordered solid solutions and similar peculiarities in heterogeneous structures. Low Temp. Phys. Vol. 34, pp. 829 - 841.

Gurevich V.L., Parshin D.A. \& Schrober H.R. (2003) Anharmonicity, vibrational instability, and the Boson peak in glasses. Phys.Rev. B., Vol. 67. pp. 094203-01 - 094203-10.

Haydock R., Heine V. \& Kelly M.J. (1972) Electronic structure based on the local atomic environment for tight-binding bands. Journ. of Phys. C. Vol. 5, pp. 2845-2858.

Hehlen B., Courtens E., Vacher R., Yamanaka A., Kataoka M. \& Inoue K. (2000) HyperRaman Scattering Observation of the Boson Peak in Vitreous Silica, Phys. Rev. Lett., Vol. 84, pp. 5355 - 5358

Ivanov M.A. (1970) Dynamics of quasi-local vibrations at a high concentration of impurity centers. Fiz. Tv. Tela, Vol. 12, pp. 1895-1905 (in Russian).

Ivanov M.A. \& Skripnik Yu. V. (1994) On the convergence and pair corrections to the coherent potential. Fiz. Tv. Tela, Vol. 36, pp. 94-105 (in Russian).

Kagan Yu. \& Iosilevsky Ya. (1962) Mossbauer effect for an impurity nucleus in a crystal. Zh. Eksp. Teor. Fiz. Vol. 44, pp. 259-272 (in Russian).

Klinger M.I. \& Kosevich A.M. (2001) Soft-mode-dynamics model of acoustic-like high-frequency excitations in boson-peak spectra of glasses. Phys. Lett. A, Vol. 280, pp. 365 - 370.

Klinger M.I. \& Kosevich A.M. (2002) Soft-mode dynamics model of boson peak and high frequency sound in glasses: "inelastic" Ioffe-Regel crossover and strong hybridization of excitations. Phys. Lett. A, Vol. 295, pp. 311 - 317.

Koestler L., Wurdack S., Dietsche W. \& Kinder H. (1986). Phonon Spectroscopy of Adsorbed $\mathrm{H}_{2} \mathrm{O}$ Molecules, In: Phonon scattering in condensed matter $V$, Meissner M., Pohl R.O. (Ed), 171 -173, ISBN 0-387-17057-X Springer-Verlag, New York, Berlin, Heidelberg.

Kosevich A.M. (1999). The Crystal Lattice (Phonons, Solitons, Dislocations), WILEY-VCH Verlag Berlin $\mathrm{GmBH}$, Berlin

Kosevich A.M., Gospodarev I.A., Grishaev V.I., Kotlyar O.V., Kruglov V.O., Manzheliŭ E.V., Syrkin E.S. \& Feodosyev S.B. (2007) Peculiarities of transformations of local levels into impurity zone on example of disordered solid solutions $\mathrm{Ag}_{1-\mathrm{p}} \mathrm{Al}_{\mathrm{p}}$. Journal of Experimental and Theoretical Physics, Vol. 132, pp. 11 - 18.

Kosevich Yu.A., Feher A. \& Syrkin E.S. (2008) Resonance absorption, reflection, transmission of phonons and heat transfer through interface between two solids. Low Temp. Phys. Vol. 34, pp. 725 - 733.

Kovalev O.V. (1961) Irreducible Representations of the Space Groups, Gordon and Breach, New York 1965, Ukrainian Academy of Sciences Press, Kiev. 
Lang N.D. (1986) Theory of single-atom imaging in the scanning tunneling microscope. Phys. Rev. Lett., Vol. 56, pp. 1164 - 1167.

Leibfried G. (1955) Gittertheorie der Mechanischen und thermishen Eigenschaften der Kristalle. Springer-Verlag, Berlin, Göttingen, Heidelberg.

Lifshitz I.M., Gredeskul S.A. \& Pastur L.A. (1988) Introduction to the Theory of Disordered Systems, Wiley, New York.

Lifshits I.M. (1952a) About one problem of perturbation theory in quantum statistics. Usp. Mat. Nauk, Vol. 7, pp. 171 - 180 (in Russian).

Lifshits I.M. (1952b) About heat properties of chain and layered structures. Journal of Experimental and Theoretical Physics, Vol. 22, pp.475 - 478.

Liu Z., Zhang X., Mao Y., Zhu Y.Y., Yang Z., Chan C. T. \& Sheng P. (2000) Locally resonant sonic materials. Science, Vol. 289, pp. $1734-1736$.

Manzhelii V.G., Chausov V.P. \& Kovalenko S.I. (1970) Heat capacity of solid solutions ArXe in low temperature. Fiz. Tv. Tela., Vol. 12, pp. 2764 - 2766.

Maradudin A.A., Montroll E.W., Weiss G.N. \& Ipatova I.P. (1982) Lattice Dynamics and Models of Interatomic Forces, Springer Series in Solid State Sciences. Springer-Verlag, Berlin, Heidelberg, New York.

Moncton D.E., Axe J.D. \& Di Salvo F.J. (1975) Study of Superlattice Formation in $2 \mathrm{H}-\mathrm{TaSe}_{2}$ by Neutron Scattering. Phys. Rev. Lett. , Vol. 34, pp. $734-737$.

Peresada V.I. (1968) New computational method in theory of crystal lattice. In Condensed Matter Physics( in Russian), 172 - 210, FTINT AN UkrSSR, Kharkov, Ukraine.

Peresada V.I. \& Tolstoluzhskij V.P. (1970) On the influence of impurity atoms on the thermodynamic properties of the face centered cubic lattice. Preprint (in Russian), FTINT AN UkrSSR (Institute for Low Temperature Physics and Engineering, National Academy of Sciences of Ukraine), Kharkov, Ukraine.

Peresada V.I. \& Tolstoluzhski V.P. (1977) Low-temperature heat capacity of FCC crystal containing substitution impurities. Fiz. Nizk. Temp. (Sov. J. Low Temp. Phys.), Vol. 3, pp. $788-800$.

Peresada V.I., Afanas'ev V.N., \& Borovikov V.S. (1975) Calculation of the distribution function for single-magnon excitation in an antiferromagned. Fiz. Nizk. Temp. (Sov. J. Low Temp. Phys.), Vol. 1, pp. 461 - 472.

Rufflé B., Guimbretière G., Courtens E., Vacher R. \& Monaco G. (2006) Glass-Specific Behavior in the Damping of Acousticlike Vibrations. Phys. Rev. Lett., Vol. 96, pp. 045502-1 - 045502-4.

Schrimacher W., Diezemann G., \& Ganter C. (1998) Harmonic Vibrational Excitations in Disordered Solids and the "Boson Peak". Phys. Rev. Lett., Vol. 81, pp. 136-139.

Shklyarevski O.I, Yanson I.K. \& Lysykh A.A. (1975). The inelastic tunneling effect in thin films of solidified gases Fiz. Nizk. Temp. (Sov. J. Low Temp. Phys.), Vol. 1, pp. 502 - 505.

Shkorbatov A.G., Feher A. \& Stefanyi P. (1996) Ballistic Landauer-tipe thermal conductivity of a dielectric point contact. Physica B, Vol. 218, pp. 242 - 244.

Shkorbatov A.G., Stefanyi P., Bystrenova E. \& Feher A. (1998). Phonon transport in pressure-made point contacts.J. Phys.: Condens. Matter, Vol. 10, pp. 8313 - 8326.

Syrkin E.S. \& Feodosyev S.B. (1982) The contribution of optical vibrations in the lowtemperature heat capacity of layered crystals. Low Temp. Phys. Vol. 8, pp. 760 - 764.

Tarasov V.V. (1950) Theory of heat capacity of chain and layered structures. Russian Journal of Physical Chemistry A, Vol. 24, pp. $111-128$.

Wakabayashi N., Smith H.G. \& Shanks R. (1974) Two dimensional Kohn anomaly in $\mathrm{NbSe}_{2}$. Phys. Lett. A, Vol. 5, pp. 367 - 368. 


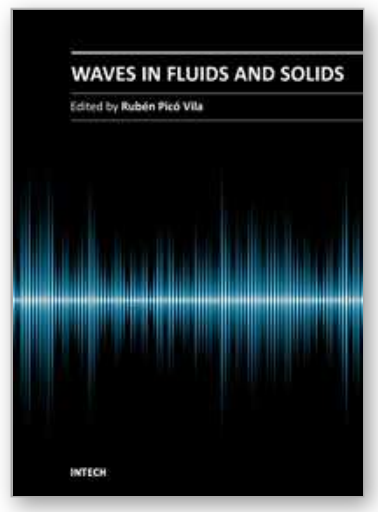

\author{
Waves in Fluids and Solids \\ Edited by Prof. Ruben Pico Vila
}

ISBN 978-953-307-285-2

Hard cover, 314 pages

Publisher InTech

Published online 22, September, 2011

Published in print edition September, 2011

Acoustics is an discipline that deals with many types of fields wave phenomena. Originally the field of Acoustics was consecrated to the sound, that is, the study of small pressure waves in air detected by the human ear. The scope of this field of physics has been extended to higher and lower frequencies and to higher intensity levels. Moreover, structural vibrations are also included in acoustics as a wave phenomena produced by elastic waves. This book is focused on acoustic waves in fluid media and elastic perturbations in heterogeneous media. Many different systems are analyzed in this book like layered media, solitons, piezoelectric substrates, crystalline systems, granular materials, interface waves, phononic crystals, acoustic levitation and soft media. Numerical methods are also presented as a fourth-order Runge-Kutta method and an inverse scattering method.

\title{
How to reference
}

In order to correctly reference this scholarly work, feel free to copy and paste the following:

Alexander Feher, Eugen Syrkin, Sergey Feodosyev, Igor Gospodarev, Elena Manzhelii, Alexander Kotlar and Kirill Kravchenko (2011). The Features of Low Frequency Atomic Vibrations and Propagation of Acoustic Waves in Heterogeneous Systems, Waves in Fluids and Solids, Prof. Ruben Pico Vila (Ed.), ISBN: 978-953307-285-2, InTech, Available from: http://www.intechopen.com/books/waves-in-fluids-and-solids/the-featuresof-low-frequency-atomic-vibrations-and-propagation-of-acoustic-waves-in-heterogeneous-s

\section{INTECH}

open science | open minds

\section{InTech Europe}

University Campus STeP Ri

Slavka Krautzeka 83/A

51000 Rijeka, Croatia

Phone: +385 (51) 770447

Fax: +385 (51) 686166

www.intechopen.com

\section{InTech China}

Unit 405, Office Block, Hotel Equatorial Shanghai

No.65, Yan An Road (West), Shanghai, 200040, China

中国上海市延安西路65号上海国际贵都大饭店办公楼405单元

Phone: +86-21-62489820

Fax: $+86-21-62489821$ 
(C) 2011 The Author(s). Licensee IntechOpen. This chapter is distributed under the terms of the Creative Commons Attribution-NonCommercialShareAlike-3.0 License, which permits use, distribution and reproduction for non-commercial purposes, provided the original is properly cited and derivative works building on this content are distributed under the same license. 